\title{
Signs and symptoms, evaluation, and management of genitourinary tract consequences of premature ovarian insufficiency
}

\author{
Anna Calik-Ksepka, Monika Grymowicz, Ewa Rudnicka, Jolanta Skórska, Paulina Machura, \\ Wojciech Pięta, Roman Smolarczyk
}

Department of Gynaecological Endocrinology, Medical University of Warsaw, Warsaw, Poland

\begin{abstract}
Premature ovarian insufficiency (POI) occurs in $1 \%$ of women under 40 years old. Hypoestrogenism associated with this condition may result in vaginal atrophy and urine incontinence, called genitourinary syndrome. The symptoms include: vaginal dryness, irritation, dyspareunia, and dysuria. There is relative lack of studies on the occurrence and treatment of genitourinary problems in women with POI. Prevalence rates vary from 17 to $54 \%$ depending on cause, duration of oestrogen depletion, and the treatment used. Patients with POI gain lower scores in tests measuring vaginal health or sexual function in comparison to healthy peers. Hormonal treatment in premature ovarian insufficiency is recommended until the natural age of menopause. The vaginal route of oestrogen administration is supposed to be the criterion standard in treating genitourinary symptoms. Androgen supplementation is not routinely recommended.
\end{abstract}

Key words: premature ovarian insufficiency, genitourinary syndrome, oestrogen therapy.

\section{Introduction}

Premature ovarian insufficiency $(\mathrm{POI})$ is a condition characterised by sex steroid deficiency, elevated gonadotropin follicle-stimulating hormone $(\mathrm{FSH})>25 \mathrm{ml} /$ IU four weeks apart, and oligo/amenorrhoea for more than four months in women below 40 years old. The prevalence of $\mathrm{POI}$ varies from $0.01 \%$ in women under 20 years of age to $1 \%$ in patients under 40 years of age.

Studies on menopausal patients show that depletion of ovarian function and lack of oestradiol may result in sequelae of vaginal atrophy and urine incontinence, called genitourinary (GU) syndrome. There are few trials on urogynaecological consequences in young patients with $\mathrm{POI}$, and therefore most evidence is derived from studies in postmenopausal women of older age.

\section{Signs and symptoms}

Prolonged hypoestrogenism may lead to reduced transudation through the vaginal epithelium and diminished cervical gland secretion. Signs of vaginal atrophy are dryness, itching, burning, irritation, discharge, and dyspareunia. Those symptoms may occur gradually or suddenly and usually are progressive with the time of oestradiol deficiency. However, not all women with diminished ovarian function will suffer from genitouri- nary (GU) symptoms [1]. GU is associated with sexual disturbances, and lower urinary tract symptoms, such as urgency, nocturia, and dysuria. Urine incontinence and recurrent infection are reported more frequently after menopause [2].

\section{Incidence}

Genitourinary syndrome of menopause affects more than $50 \%$ of postmenopausal women. Data may be underestimated; the real prevalence is thought to be higher due to sexual embarrassment and the sensitive nature of the problem [3]. The exact prevalence rate of $\mathrm{GU}$ symptoms in women with spontaneous POI is not known. Data from an observational study, assessing GU symptoms in 31 women with POI, revealed that 54\% suffered from symptoms of vulvovaginal atrophy (dyspareunia, itching, burning sensation) and $42 \%$ had urinary tract symptoms (dysuria, urinary frequency, mild urinary incontinence) [4]. In women with iatrogenic POI after breast cancer treatment the prevalence of moderate to severe dyspareunia was $42 \%$, and the incidence of vaginal dryness was $49 \%$ [5]. Data from patients after bilateral oophorectomy showed a $24 \%$ prevalence rate for vaginal dryness, and $17 \%$ for dyspareunia. Loss of libido was reported by $22 \%$ of women without any hormonal treatment [6]. 


\section{Aetiopathogenesis}

Oestrogen receptors are localised in the vagina, vulva, vestibule, labia majora/minora, and bladder trigone in high concentration. These parts are sensitive biological markers of serum hormonal levels [7]. Oestradiol influences synthesis of local peptides, which is of great value for vaginal microflora [8]. Moreover, oestrogens promote production of antimicrobial peptides and exfoliation of infected cells. Hypoestrogenic state is correlated with increased incidence of urinary tract infections after menopause.

Vaginal disturbances like atrophy or infections can be assessed by collecting hormonal cytology, $\mathrm{pH}$ measurement, amine test, bacterioscopy, and culture for bacteria and fungi. Vaginal health index (VHI) includes the assessment of overall elasticity, fluid secretion, $\mathrm{pH}$, epithelial integrity, and dankness [9]. Bonetti-Pinto et al. found no statistical differences in cytology, $\mathrm{pH}$, or microflora in women with POI compared to healthy controls. In both groups VHI was trophic, although POI patients were found to have statistically lower $\mathrm{VHI}$ scores than controls [9]. The VHI scores were lower than in the control group mainly because of less vaginal discharge and friable appearance with the presence of petechiae [10].

Progesterone in the urinary tract acts mainly by inducing beta-adrenergic receptor formation, alpha-adrenergic antagonism, and anticholinergic effect. Progesterone increases bladder capacity and dilates the urethra through relaxation of smooth muscles [11].

Symptomatology of POI depends not only of oestrogen and progesterone serum levels but is also connected with decline in ovarian testosterone production. In premenopausal women androgens are synthetized in $50 \%$ by ovaries and $50 \%$ by adrenal glands. Androgens act centrally through dopamine and peripherally through nitric oxide pathways, and they may result in improved libido and overall quality of life [12-14].

\section{Sexual function}

Sexual function (SF) in women is under modulation of oestrogen influencing lubrication and androgen stimulating desire and arousal. A case-controlled study on sexuality in young women with spontaneous POI reported normal sexual function despite lower testosterone serum levels than in the control group. However, lower serum total testosterone levels were correlated with the decreased Derogatis Interview for Sexual Functioning (DISF) composite score [15].

Pacello et al. assessed sexual function of 36 women with POI using Female Sexual Function Index (FSFI). Women with $\mathrm{POI}$ showed worse sexual performance with complaints about lubrication and pain during intercourse. No differences in desire, arousal, orgasm, or satisfaction in FSFI questionnaire were found between groups. Use of systemic oestrogen in the POI group was not enough to improve sexual function, despite normal vaginal tropism assessed with $\mathrm{VHI}$ measurements [10].

Yela et al. examined 80 women with $\mathrm{POI}$ regarding their sexual function and quality of life (QoL). Psychological domains like orgasm, arousal, and sexual satisfaction were correlated with stronger impact on QoL than physical disturbances like pain or lubrication [16]. Loss of gonadal function impairs SF and QoL regardless of hormonal treatment. Early and unexpected infertility has a negative impact on sexuality by decline in self-confidence and interpersonal relationships [16-19]. The study of Bonetti-Pinto et al. on 58 women with POI showed lower sexual function scores because of decreased arousal, lubrication, orgasm, and satisfaction and higher incidence of dyspareunia. The greatest differences were shown in decline of arousal and desire, and less in dyspareunia and lubrication [20].

\section{Management \\ Hormone replacement treatment}

Hormone replacement therapy (HRT), both systemic and topical, is implemented to treat genitourinary symptoms. According to the National Institute of Health $(\mathrm{NIH})$, the European Menopause and Andropause Society (EMAS), and the International Menopause Society (IMS), oestrogen treatment is recommended for vaginal dryness. The EMAS recommends using both systemic and topical oestrogen initially if symptoms are severe, followed by just local treatment [14, 21-23].

Early detection and individualised treatment is crucial. Oestrogen therapy is the most commonly prescribed treatment. Many different formulations are available, from pessaries, creams, and rings, to tablets. For the majority of treated women, both local and systemic HRT seem to be effective in relieving genitourinary symptoms. The vaginal route of administration delivers oestrogen locally with little or no influence on endometrium and systemic receptors. That is why no progestins are obligatory during such application [8]. According to the Cochrane review, all forms of vaginal oestrogen are equally effective [24]. Hormonal treatment reduces vaginal atrophy symptoms and corrects the physical changes, and restores the cytology, $\mathrm{pH}$, and vascularity of the vagina [2]. However, the longer hypoestrogenism proceeds, the more difficult atrophy may be to treat [2].

There is lack of randomised controlled trials assessing the use of HRT in the treatment of genitourinary syndrome in women with premature ovarian insufficiency. In the Piccioni study, systemic HRT in POI patients with vulvovaginal atrophy and urinary tract 
symptoms resolved atrophy and GU dysfunction in 53\% of the treated women after five weeks of therapy [4].

Oestrogen treatment does not change urethral pressure nor cystometric parameters during urodynamic tests, regardless of the route of oestradiol administration. Karram et al., in a study on a group of six POI patients using vaginal oestrogen cream, showed differences in pressure transmission ratio to the proximal and mid-urethra. Nevertheless, the authors concluded that hypoestrogenaemia alone, apart from age or other factors causing urine loss, has a minimal impact on incontinence [25].

Local oestrogen therapy reduces the rate of recurrent urinary infections $[2,26]$. However, Burton et al., assessing 217 women with $\mathrm{POI}$ on continuous systematic oestrogen and cyclic progestin therapy, found minimal hormonal effect on the genitourinary tract. Although there were more complaints of urgency during luteal phase, the rate of stress incontinence was not changed [27].

The main goal for replacement therapy is to mimic normal ovarian function. Some authors suggest a beneficial effect of adding testosterone during oestrogen hormonal therapy [14]. Women with sexual disturbances, who, prior to diagnosis of POI, had satisfying sexual life and suffer from deterioration in desire may benefit from short-term use of testosterone patches of $300 \mu \mathrm{g}$ daily. However, the long-term consequences are still unknown, and androgen supplementation in patients with premature ovarian insufficiency remains controversial. Currently, diagnosis or treatment of testosterone deficiency in women with POI is not recommended [14].

Unfortunately, a recent study showed that more than half (52\%) of young women with POI either never take HRT, start HRT many years after their diagnosis, and/or discontinue HRT use prior to 45 years of age $[2,7]$.

\section{Non-hormond treatment}

Non-hormonal treatment can be used additionally to hormones. It is safe even in women who have contraindications to hormone therapy, like in the group of iatrogenic $\mathrm{POI}$ patients with oestrogen-dependent cancer history [1]. For women who cannot use hormonal treatment or those who prefer non-medical interventions, low-risk approaches may be of value and may be more acceptable. Non-hormonal methods include vaginal lubricants, moisturisers, laser treatment, ospemifene, and lifestyle modifications. Pelvic floor physical therapy can be recommended in order to correct pelvic floor muscle dysfunction [3, 28].

Local treatment with lubricants and moisturisers is often the first-line treatment. Lubricants are water-based or silicon-based preparations that replace normal vaginal secretion. Moisturisers act longer than lubricants and some contain mucoadhesive polymers, hyaluronic acid, or isoflavones. Unfortunately, for many women the use of lubricants or moisturisers does not adequately ameliorate the symptoms.

A new treatment modality, intravaginal laser therapy, is a promising tool to alleviate genitourinary syndrome. A recently performed meta-analysis suggests that laser therapy is a very promising and safe treatment option in postmenopausal women with GS [29]. However, the quality of the body of evidence is "low" or "very low", and because there is a lack of well-designed randomised trials and because the placebo effect cannot be ruled out, such treatment cannot be proposed as evidence-based practice [29].

Ospemifene is a selective oestrogen receptor modulator (SERM) approved for the treatment of urogenital symptoms at menopause. It is administered daily, orally, during meals. Ospemifene is effective not only in treating vulvovaginal atrophy and dyspareunia, but also some research showed that it decreases the incidence of stress urine incontinence and urinary tract infections [30]. Treatment with ospemifene increases risk of thromboembolism but probably has a good safety-profile regarding breast-related issues. Ospemifene effectiveness has not been studied in women with POI.

Sexual dysfunction is often multifactorial, and not only oestrogen deprivation but also complex psychological aspects concerning self-assessment and fertility problems may add to patients' uncertainty and distress [12]. Women with POI often need counselling. Emotional support from professionals and family plays an essential role in the management of patients with POI [19].

\section{Conclusions}

The group of patients with premature ovarian insufficiency is under-researched in the context of genitourinary symptoms, and the few available studies are performed on small groups of women with POI. The young age of patients with POI probably makes them less susceptible to urine incontinence; however, more research is needed to investigate further this issue. The sensitive nature of the problems with vaginal atrophy, sexual dysfunction, or urine incontinence may cause underestimation of the problem. The mainstay of the therapy of genitourinary syndrome is oestrogen replacement, mostly administered locally. There is a need to study real differences between the genitourinary symptoms in women of postmenopausal age and younger patients with $\mathrm{POI}$ and to design the most efficient treatment options.

\section{Disclosure}

The authors report no conflict of interest. 


\section{References}

1. Simon J. Non-estrogen based treatments for vaginal atrophy. Maturitas 2015; 81: 106

2. Kokot-Kierepa M, Bartuzi A, Kulik-Rechberger B, et al. Local estrogen therapy - clinical implications - 2012 update. Ginekol Pol 2012; 83: 772-777.

3. Gandhi J, Chen A, Dagur G, et al. Genitourinary syndrome of menopause: an overview of clinical manifestations, pathophysiology, etiology, evaluation, and management. Am J Obstet Gynecol 2016; 215: 704-711.

4. Piccioni P, Scirpa P, D'Emilio I, et al. Hormonal replacement therapy after stem cell transplantation. Maturitas 2004; 49: 327-333.

5. Leining MG, Gelber S, Rosenberg R, et al. Menopausal-type symptoms in young breast cancer survivors. Ann Oncol 2006; 17: 1777-1782.

6. Madalinska JB, van Beurden M, Bleiker EM, et al. The impact of hormone replacement therapy on menopausal symptoms in younger highrisk women after prophylactic salpingo-oophorectomy. J Clin Oncol 2006; 24: 3576-3582.

7. Palacios S, Mejía A, Neyro JL. Treatment of the genitourinary syndrome of menopause. Climacteric 2015; 18 (Suppl 1): 23-29.

8. Samsioe G. Estrogen based treatments for genitourinary. Maturitas 2015; 81: 106

9. Bonetti-Pinto CL, Giraldo PC, Pacello PCC, et al. Vaginal epithelium and microflora characteristics in women with premature ovarian failure under hormone therapy compared to healthy women. Arch Gynecol Obstet 2015; 292: 159-164.

10. Pacello PCC, Yela DA, Rabelo S, et al. Dyspareunia and lubrication in premature ovarian failure using hormonal therapy and vaginal health. Climacteric 2014; 17: 342-347.

11. Swift SE, Ostergard DR. Effects of progesterone on the urinary tract. Int Urogynecol J 1993; 4: 232-236.

12. Graziottin A. Menopause and sexuality: key issues in premature menopause and beyond. Ann N Y Acad Sci 2010; 1205: 254-261.

13. Sullivan SD, Sarrel PM, Nelson LM. Hormone replacement therapy in young women with primary ovarian insufficiency and early menopause. Fertil Steril 2016; 106: 1588-1599.

14. European Society for Human Reproduction and Embryology (ESHRE) Guideline Group on POI, Webber L, Davies M, Anderson R, et al. ESHRE Guideline: management of women with premature ovarian insufficiency. Hum Reprod 2016; 31: 926-937.

15. Kalantaridou SN, Vanderhoof VH, Calis KA, et al. Sexual function in young women with spontaneous $46, \mathrm{XX}$ primary ovarian insufficiency. Fertil Steril 2008; 90: 1805-1811.

16. Yela DA, Soares PM, Laguna Benetti-Pinto C. Influence of Sexual Function on the Social Relations and Quality of Life of Women with Premature Ovarian Insufficiency. Rev Bras Ginecol Obs 2018; 40: 66-71.

17. Nelson LM. Clinical practice. Primary ovarian insufficiency. N Engl J Med 2009; 360: 606-614.

18. Orshan SA, Furniss KK, Forst C, et al. The lived experience of premature ovarian failure. J Obstet Gynecol Neonatal Nurs 2001; 30: 202-208.

19. Rafique S, Sterling EW, Nelson LM. A new approach to primary ovarian insufficiency. Obstet Gynecol Clin North Am 2012; 39: 567-586.

20. Benetti-Pinto CL, Soares PM, Giraldo HP, et al. Role of the different sexuality domains on the sexual function of women with premature ovarian failure. J Sex Med 2015; 12: 685-689.

21. NIH. NIH State-of-the-Science Conference Statement on management of menopause-related symptoms. NIH Consens State Sci Statements 2005; 22: 1-38.

22. Skouby SO, Al-Azzawi F, Barlow D, et al. Climacteric medicine: European Menopause and Andropause Society (EMAS) 2004/2005 position statements on peri- and postmenopausal hormone replacement therapy. Maturitas 2005; 51: 8-14.

23. Sturdee DW, Panay N, International Menopause Society Writing G. Recommendations for the management of postmenopausal vaginal atrophy. Climacteric 2010; 13: 509-522.

24. Suckling J, Lethaby A, Kennedy R. Local oestrogen for vaginal atrophy in postmenopausal women. Cochrane Database Syst Rev 2006: CD001500.

25. Karram MM, Yeko TR, Sauer MV, Bhatia NN. Urodynamic changes following hormonal replacement therapy in women with premature ovarian failure. Obs Gynecol 1989; 74: 208-211.
26. Hirschberg AL. Estrogens and urinary tract infections. Maturitas 2015; 81: 110.

27. Burton G, Cardozo LD, Abdalla $\mathrm{H}$, et al. The hormonal effects on the lower urinary tract in 282 women with premature ovarian failure. Neurourol Urodyn 1992; 10: 318-319.

28. Faubion SS, Sood R, Kapoor E. Genitourinary Syndrome of Menopause: Management Strategies for the Clinician. Mayo Clin Proc 2017; 92: 1842-1849.

29. Pitsouni E, Grigoradis T, Falagas ME, et al. Laser therapy for the genitourinary syndrome of menopause. A systemic review and meta-analysis. Maturitas 2017; 103: 78-88.

30. Bachmann GA, Komi JO, Ospemifene Study Group. Ospemifene effectively treats vulvovaginal atrophy in postmenopausal women: results from a pivotal phase 3 study. Menopause 2010; 17: 480-486. 